\title{
SUR LA THEORIE SPECTRALE LOCALE ET LIMITE DES NILPOTENTS
}

\author{
MOSTAFA MBEKHTA
}

(Communicated by Palle E.T. Jorgensen)

\begin{abstract}
Résumé. Dans ce travail, nous donnons une condition nécessaire et suffisante pour qu'un opérateur fermé dans un espace de Banach admette la propriété de l'extension unique. Pour cela nous introduisons la notions du coeur analytique d'un opérateur. Nous établissons plusieurs propriétés des opérateurs dont le coeur analytique est réduit à zéro (l'ensemble de ces opérateurs est noté $\mathscr{K} \mathscr{d}_{0}(H)$ où $H$ est un Hilbert), notamment nous montrons que si $T \in \mathscr{K} \mathscr{L}_{0}(H)$ alors $0 \in \sigma\left(T_{\mid M}\right)$ pour tout sous-espace invariant $M \neq\{0\}$. En particulier $\sigma_{p}(T) \subseteq\{0\}, \sigma(T)$ est connexe et contient zéro et si $T$ est décomposable alors $T$ est quasinilpotent. Nous montrons aussi que l'ensemble $(\mathscr{K} \mathscr{A}(H))$ des opérateurs $T$ tels que $T, T^{*} \in \mathscr{K} \mathscr{Q}_{0}(H)$ est inclus strictement dans l'adhérence des nilpotents $(\overline{\mathscr{N}})$. Et pour tout $T \in \overline{\mathscr{N}}$ et $\varepsilon>0$, il existe $K_{\varepsilon}$ opérateur compact tel que $\left\|K_{\varepsilon}\right\|<\varepsilon$ et $T+K_{\varepsilon} \in \mathscr{H} \mathscr{A}(H)$.
\end{abstract}

\section{INTRODUCTION}

Dans ce travail nous donnons une condition nécessaire et suffisante pour qu'un opérateur fermé dans un espace de Banach admette la propriété de l'extension unique (S.V.E.P.). Il est facile de voir que le spectre ponctuel d'un opérateur qui n'admet pas cette propriété a un intérieur non vide. La réciproque n'est pas vraie en général. Cependant on montre (Théorème 4.2) que: "si $A-\lambda_{0} I$ est pseudo-Fredholm (classe d'opérateurs qui contient les semi-Fredholm; cf. [17]) et $\lambda_{0}$ est à l'intérieur du spectre ponctuel de $A$ lors $A$ n'a pas la propriété de l'extension unique." Ce dernier résultat est une généralisation de [12]. Pour cela nous introduisons la notion du coeur analytique d'un opérateur $A$, noté $K(A)$.

Dans $\S 2$, nous étudions l'ensemble des opérateurs $T$, bornés dans un Hilbert $H$, tel que $K(T)=\{0\}$. Nous notons cet ensemble $\mathscr{H} \mathscr{A}_{0}(H)$ et nout montrons que si $T \in \mathscr{K} \mathscr{A}_{0}(H)$ alors, $0 \in \sigma\left(T_{\mid M}\right)$ pour tout sous-espace invariant $M \neq$ $\{0\}$. En particulier $\sigma_{p}(T) \subseteq\{0\}$ et $\sigma(T)$ est connexe et contient zéro.

La Proposition 2.6, montre que si $T \in \mathscr{K} \mathscr{A}_{0}(H)$ alors $\sigma(T)=\sigma_{\omega}(T)$ où $\sigma_{\omega}(T)$ est le spectre de Weyl de T. Nous montrons aussi que si $T \in \mathscr{K}^{\mathscr{L}} \mathscr{Q}_{0}(H)$

Received by the editors January 25, 1989.

1980 Mathematics Subject Classification (1985 Revision). Primary 47A05; Secondary 47B40.

Key words and phrases. Spectre local, propriété de l'extension unique, adhérence des nilpotents, opérateur pseudo-Fredholm. 
alors

$$
\{S \in B(H) ; S \text { est quasi-similaire à } T\} \subseteq \mathscr{K}_{0}(H) .
$$

Nous terminons cette section par une caractérisation du spectre d'un opérateur appartenant à $\mathscr{K} \mathscr{A}_{0}(H)$.

Dans $\S 3$, on montre que l'ensemble des opérateurs $T$ tels que $K(T)=$ $K\left(T^{*}\right)=\{0\} \quad$ (qu'on note $\mathscr{K} \mathscr{A}(H)$ ) est inclus strictement dans l'adhérence des nilpotents $(\overline{\mathscr{N}})$, et contient $\mathscr{Q}_{\text {af }} \cap \mathscr{Q}_{\text {af }}^{*}$.

Nous conjecturons que:

$$
\mathscr{K} \mathscr{A}(H)=\mathscr{Q}_{\text {af }} \cap \mathscr{Q}_{\text {af }}^{*}
$$

(où $\mathscr{Q}_{\text {af }}$ est l'ensemble des opérateurs bornés transformation quasi-affine d'opérateurs quasi-nilpotents et $T \in \mathscr{Q}_{\text {af }}^{*}$ si $T^{*} \in \mathscr{Q}_{\text {af }}$ ). Par ailleurs nous démontrons que: pour tout $T \in \overline{\mathscr{N}}$ et $\varepsilon>0$, il existe $K_{\varepsilon}$ opérateur compact tel que $\left\|K_{\varepsilon}\right\|<\varepsilon$ et $T+K_{\varepsilon} \in \mathscr{K} \mathscr{A}(H)$.

Dans $\S 4$, nous démontrons la conjecture $\left(\mathscr{K} \mathscr{A}(H)=\mathscr{Q}_{\text {af }} \cap \mathscr{Q}_{\text {af }}^{*}\right)$ dans le cas des opérateurs pseudo-Fredholm.

\section{ThÉORIE SPECTRALE LOCAL ET COEUR ANALYTIQUE}

Dans cette section $X$ désigne un espace de Banach et $A$ un opérateur fermé de domaine $D(A)$ et d'image $R(A)$ dans $X$. Notons $N(A), \sigma(A)$ et $\rho(A)$ respectivement le noyau, le spectre et l'ensemble résolvant de $A$.

On appelle coeur analytique de $A$, le sous-espace $K(A)$ de $X$ défini par:

$$
\begin{aligned}
& K(A)=\left\{u \in X ; \exists a>0,\left\{v_{n}\right\}_{n>0} \subset D(A) \text { tels que (1) } v_{0}=u \text { et } A v_{n+1}=v_{n}\right. \\
& \text { (2) } \left.\left\|v_{n}\right\| \leq a^{n}\|u\| \forall n \geq 0\right\} \text {. }
\end{aligned}
$$

Notons aussi $H_{0}(A)=\left\{u \in D^{\infty}(A) ; \lim _{n \rightarrow \infty}\left\|A^{n} u\right\|^{1 / n}=0\right\}$, où $D^{\infty}(A)=$ $\cap_{n \geq 0} D\left(A^{n}\right)$.

Soit $T$ borné alors $T \in \mathscr{Q}_{\text {af }}$ s'il existe $Q$ quasi-nilpotent $(\sigma(Q)=\{0\})$ et $S$ quasi-affinité $\left(N(S)=N\left(S^{*}\right)=\{0\}\right)$ tels que $S T=Q S$.

Remarque 1.1 . (a) $K(A)$ et $H_{0}(A)$ sont des sous-espaces de $X$ non nécéssairement fermés tels que

$$
A(K(A) \cap D(A))=K(A) \quad \text { et } \quad A\left(H_{0}(A)\right) \subseteq H_{0}(A) .
$$

(b) Si $A$ est borné et $A \in \mathscr{Q}_{\text {af }}$ alors $K(A)=\{0\}$.

(c) $H_{0}(A)=X$ si et seulement si $A$ est quasi-nilpotent.

Démonstration. (a) Voir $[15,16]$.

(b) Soit $u \in K(A)$ et $\left\{v_{n}\right\}, a>0$ de la définition de $K(A)$. Si $A \in \mathscr{Q}_{\text {af }}$ alors $\forall n \geq 0, S A^{n}=Q^{n} S . \quad\|S u\|=\left\|S A^{n} v_{n}\right\|=\left\|Q^{n} S v_{n}\right\| \leq\left\|Q^{n}\right\|\|S\| a^{n}\|u\| \rightarrow 0$ quand $n \rightarrow \infty$ donc $\|S u\|=0$ et comme $N(S)=\{0\}$ on conclu que $u=0$.

(c) Voir [20, Théorème 1.5]. 
Soit $u \in X$ fixé, notons $\delta_{A}(u)$ l'ensemble des $\mu \in \mathbb{C}$ tel que, il existe un voisinage $\mathscr{V}_{\mu}$ de $\mu$ dans $\mathbb{C}$ et une fonction $f: \mathscr{V}_{\mu} \rightarrow D(A)$ analytique (dans $\mathscr{V}_{\mu}$ ) vérifiant:

$$
(\lambda I-A) f(\lambda)=u \quad \forall \lambda \in \mathscr{V}_{\mu} .
$$

$\delta_{A}(u)$ est appelé l'ensemble résolvant local de $A$ en $u$, son complémentaire dans $\mathbb{C}$, qu'on note $\gamma_{\hat{A}}(u)$ est le spectre local de $A$ en $u$ (voir $[4,12,19,20]$ ).

Définition 1.2. Soit $A$ un opérateur fermé dans un espace de Banach $X$ et $\mu \in \mathbb{C}$, on dit que $A$ admet la propriéte de l'extension unique en $\mu$ (et on note $A$ a la S. V. E. P. en $\mu$ ) si $f=0$ est la seule solution analytique dans un voisinage de $\mu$ de l'équation: $(\lambda I-A) f(\lambda)=0$.

On dira aussi que $A$ a la S. V. E. P. si $\forall \lambda \in \mathbb{C}, A$ a la S.V.E.P. en $\lambda$.

Proposition 1.3. Soit $A$ un opérateur fermé dans un Banach $X$ on $a$ :

(a) $K(A)=\left\{u \in X ; 0 \in \delta_{A}(u)\right\}$,

(b) $H_{0}(A) \subseteq\left\{u \in X ; \gamma_{\hat{A}}(u) \subseteq\{0\}\right\}$ (on a l'égalité si $A$ a la S.V.E.P.).

Démonstration. (a) Soit $u \in K(A)$ et $a>0,\left\{v_{n}\right\}_{n \geq 0}$ provenant de la définition de $K(A)$. Alors la fonction $f: \mathscr{U}:=\left\{\lambda \in \mathbb{C} ;|\lambda|<a^{-1}\right\} \rightarrow X$ définie par $f(\lambda)=\sum_{n \geq 0} v_{n} \lambda^{n-1}$ est analytique dans $\mathscr{U}$, à valeurs dans $D(A)$ et vérifie:

$$
(\lambda I-A) f(\lambda)=u \quad \forall \lambda \in \mathscr{U}, \quad \text { donc } 0 \in \delta_{A}(u) .
$$

Réciproquement: si $u \in X$ tel que $0 \in \delta_{A}(u)$, alors par définition de $\delta_{A}(u), \exists \varepsilon$ $>0$ et $f$ analytique dans $\mathscr{U}_{\varepsilon}(0)$ voisinage de zéro, àvaleurs dans $D(A)$ vérifiant:

$$
(\lambda I-A) f(\lambda)=u \quad \forall \lambda \in \mathscr{U}_{\varepsilon}(0),
$$

$f$ étant analytique dans $\mathscr{U}_{\varepsilon}(0)$, on a:

$$
f(\lambda)=\sum_{n \geq 1} \lambda^{n-1} v_{n} \quad \forall \lambda \in \mathscr{U}_{\varepsilon}(0), \quad \text { avec } v_{n} \in D(A) .
$$

Si $a>1 / \varepsilon$ alors $\exists c>0$ tel que

$$
\left\|v_{n}\right\| \leq c a^{n-1} .
$$

De (1) et (2) on en déduit que $A v_{1}=u=v_{0}$ et $A v_{n}=v_{n-1}$, pour $n=$ $2,3, \ldots$. Si $u=0$ on prend $\forall n \geq 0, v_{n}=0$. Si $u \neq 0$ alors (3) implique,

$$
\left\|v_{n}\right\| \leq\|u\|(a+c /\|u\|)^{n} \quad \text { donc } u \in K(A) .
$$

(b) Montrons que $H_{0}(A) \subseteq\left\{u \in X ; \gamma_{A}(u) \subseteq\{0\}\right\}$. Soit $u \in H_{0}(A)$ par définition $\lim _{n \rightarrow \infty}\left\|A^{n} u\right\|^{1 / n}=0$ donc $\forall \lambda \neq 0, \Sigma_{n \geq 0} \lambda^{-n-1} A^{n} u$ est convergente. Posons $f(\lambda)=\Sigma_{n \geq 0} \lambda^{-n-1} A^{n} u$. On a $\forall \lambda \neq 0,(\lambda I-A) f(\lambda)=u$ donc $\forall \lambda \neq 0$, $\lambda \in \delta_{A}(u)$ d'où $\gamma_{A}(u) \subseteq\{0\}$.

Soit maintenant $u \in X$ tel que $\gamma_{A}(u) \subseteq\{0\}$. Si $A$ a la S. V. E. P. alors on en déduit qu'il existe $f$ analytique dans $\mathbb{C}^{*}$ à valeurs dans $D(A)$ telle que $(\lambda I-A) f(\lambda)=u$. 
On peut écrire $f(\lambda)=\Sigma_{n \geq 0} \lambda^{-n} v_{n}$ avec $v_{n} \in D(A)$ et puisque $f$ est analytique à l'infini, notons $f(\infty)=\lim _{\lambda \rightarrow \infty} f(\lambda)$. Soit $\left\{\lambda_{p}\right\}$ une suite qui tend vers l'infini quand $p$ tend vers l'infini alors $\lim _{p \rightarrow \infty} Z_{p}=\lim _{p \rightarrow \infty}\left(f\left(\lambda_{p}\right) / \lambda_{p}\right)=0$. D'autre part $A Z_{p}=-u / \lambda_{p}+f\left(\lambda_{p}\right)$.

Donc $\lim _{p \rightarrow \infty} A Z_{p}=f(\infty)$. Comme $A$ est fermé on en déduit que $f(\infty)=$ 0 par conséquent $v_{0}=0$ et $0=\lim _{\lambda \rightarrow \infty} A f(\lambda)=\lim _{\lambda \rightarrow \infty}(\lambda f(\lambda)-u)=v_{1}-u \Rightarrow$ $u=v_{1} \operatorname{donc}_{\lim _{\lambda \rightarrow \infty}} \lambda f(\lambda)=u, \lim _{\lambda \rightarrow \infty} \lambda A f(\lambda)=\lim _{\lambda \rightarrow \infty} \lambda(\lambda f(\lambda)-u)=v_{2} \Rightarrow$ $u \in D(A)$ et $A u=v_{2}$ par le même procédé on voit que $\forall n \geq 0, u \in D\left(A^{n}\right)$ et $v_{n+1}=A^{n} u$. D'autre part $\left\|\lambda^{-n} v_{n}\right\| \rightarrow 0 \Rightarrow \forall \lambda>0, \exists N>0$ tels que $\forall n>N$, on a $\left\|v_{n}\right\|<\lambda^{n}$ donc $\left\|v_{n}\right\|^{1 / n} \rightarrow 0$ et par conséquent $\left\|A^{n} u\right\|^{1 / n} \rightarrow 0$ donc $u \in H_{0}(A)$.

Théorème 1.4. Soit $A$ un opérateur fermé et $\lambda_{0} \in \mathbb{C}$; les conditions suivantes sont équivalentes:

(a) $A$ a la S.V.E.P. en $\lambda_{0}$.

(b) $K\left(\lambda_{0} I-A\right) \cap H_{0}\left(\lambda_{0} I-A\right)=\{0\}$.

Démonstration. Sans perte de généralité on supposera que $\lambda_{0}=0$.

(a) $\Rightarrow$ (b) Supposons que $A$ a la S.V.E.P. en 0 et soit $u \in K(A) \cap H_{0}(A)$; $u \in H_{0}(A) \Rightarrow \forall \lambda \neq 0, g(\lambda)=\Sigma_{n \geq 0} \lambda^{-n-1} A^{n} u$ est convergente et vérifie

$$
(\lambda I-A) g(\lambda)=u \text {. }
$$

D'autre part $u \in K(A)$ (comme dans la Proposition 1.3), $f: \mathscr{U}=\{\lambda \in \mathbb{C} ;|\lambda|<$ $\left.a^{-1}\right\} \rightarrow X$ définie par $f(\lambda)=\Sigma_{n \geq 1} \lambda^{n-1} v_{n}$ pour $|\lambda|<a^{-1}$, est analytique dans $\mathscr{U}$ à valeurs dans $D(A)$ et vérifie

$$
(\lambda I-A) f(\lambda)=u \text {. }
$$

$A$ a la S.V.E.P. en zéro; (1) et (2) entrainent que $g(\lambda)=f(\lambda)$ dans $\mathscr{U}-\{0\}$ donc $g(\lambda)$ est analytique dans tout $\mathbb{C}$, ceci n'étant possible que si $g(\lambda)$ est constante, par conséquent $(1) \Rightarrow g(\lambda)=0$ et donc $u=0$.

(b) $\Rightarrow$ (a) Soit $\mathscr{U}$ un voisinage de zéro dans $\mathbb{C}$ et $f: \mathscr{U} \rightarrow D(A)$ analytique dans $\mathscr{U}$ telle que

$$
(\lambda I-A) f(\lambda)=0 \quad \forall \lambda \in \mathscr{U} .
$$

on supposera que $\mathscr{U}=\{\lambda \in \mathbb{C} ;|\lambda|\langle r\}$ pour $r>0$. $f$ étant analytique dans $\mathscr{U}$, on peut écrire $f(\lambda)=\Sigma_{n \geq 0} \lambda^{n} u_{n}$ avec $u_{n} \in D(A)$. Si $a>1 / r$ alors $\exists c>0$ tel que

$$
\left\|u_{n}\right\| \leq c a^{n} .
$$

(3) $\Rightarrow \forall \lambda \in \mathscr{U}, \Sigma_{n \geq 0}\left(\lambda^{n+1} u_{n}-\lambda^{n} A u_{n}\right)=0$ d'où $A u_{0}=0, u_{0}=A u_{1}, \ldots, u_{n}=$ $A u_{n+1}$.

Si $\forall n \geq 0, u_{n}=0$ alors $f(\lambda)=0$. Sinon $\exists k \in \mathbb{N}$ tel que $u_{k} \neq 0$ et $A u_{k}=0$ donc pour $0 \leq j \leq k-1, u_{j}=0$. (4) implique

$$
\left\|u_{n}\right\| \leq\left\|u_{k}\right\|\left(a+c /\left\|u_{k}\right\|\right)^{n+1} \quad \forall n \geq 0,
$$


d'où $u_{k} \in K(A) \cap N(A)$; on en déduit que $\forall n \geq 0, u_{n} \in K(A) \cap N\left(A^{n}\right)$ donc $\forall n \geq 0, u_{n} \in K(A) \cap H_{0}(A)=\{0\}$ et donc $\forall n \geq 0, u_{n}=0$ par conséquent $f(\lambda)=0$. Donc $A$ a la S.V.E.P. en zéro.

Corollaire 1.5. Soit $A$ un opérateur fermé dans un Banach $X$, alors $A$ a la S.V.E.P. si et seulement si $\forall \lambda \in \mathbb{C}, K(\lambda I-A) \cap H_{0}(\lambda I-A)=\{0\}$.

Corollaire 1.6. Soit $A$ un opérateur fermé dans un Banach $X$ et supposons qu'il existe $\lambda_{0} \in \mathbb{C}$ tel que $\lambda_{0} I-A$ soit surjectif et non injectif alors $A$ n'a pas la S.V.E.P.

Démonstration. $\quad \lambda_{0} I-A$ est surjectif $\Rightarrow K\left(\lambda_{0} I-A\right)=X$. Et comme $N\left(\lambda_{0} I-A\right)$ $\neq\{0\}$ on a $K\left(\lambda_{0} I-A\right) \cap H_{0}\left(\lambda_{0} I-A\right) \neq\{0\}$.

Corollaire 1.7. Soit $A$ un opérateur fermé tel que $A$ a la S.V.E.P. alors $\lambda \in \sigma(A)$ si et seulement si $\lambda I-A$ n'est pas surjectif.

Corollaire 1.8. Soit $A$ un opérateur fermé tel que $\exists Y$ sous-espace fermé de $X$ vérifiant $A(Y \cap D(A))=Y$. Alors $N\left(\left.A\right|_{Y}\right) \neq\{0\} \Rightarrow A$ n'a pas la S.V.E.P.

Démonstration. $Y$ étant fermé et vérifiant $A(Y \cap D(A))=Y$, on en déduit que $Y \subseteq K(A)$, donc $N\left(\left.A\right|_{Y}\right) \subseteq K(A) \cap H_{0}(A)$ et par conséquent $K(A) \cap H_{0}(A) \neq$ $\{0\}$.

Proposition 1.9. Soit $T$ un opérateur borné. Alors $T \in \mathscr{Q}_{\mathrm{af}} \Rightarrow T$ a la S.V.E.P.

Démonstration. $T \in \mathscr{Q}_{\mathrm{af}} \Rightarrow K(T)=\{0\}$ (voir Remarque 1.1(b)). En outre $\forall \lambda \neq 0, N(\lambda I-T) \subseteq K(T)$ d' où on en déduit que $\sigma_{p}(T) \subseteq\{0\}$ et par conséquent $T$ a la S.V.E.P.

Proposition 1.10. Soit $A$ un opérateur fermé et $\lambda_{0} \in \mathbb{C}$. Alors $H_{0}\left(\lambda_{0} I-A\right)$ fermé dans $X \Rightarrow A$ a la S.V.E.P. en $\lambda_{0}$.

Démonstration. on supposera que $\lambda_{0}=0, H_{0}(A)$ est fermé. Soit $\hat{A}$ la restriction de $A$ à $H_{0}(A)$, d'après la Remarque 1.1(c), on en déduit que $\hat{A}$ est quasinilpotent. $K(A) \cap H_{0}(A)=K\left(A_{H_{0}(A)}\right)=K(\hat{A})=\{0\}$ car $\hat{A}$ est quasi-nilpotent, donc $K(A) \cap H_{0}(A)=\{0\}$.

Corollaire 1.11. Soit $T$ un opérateur borné dans un Hilbert $H$. Alors $T$ hyponormal $\Rightarrow T$ a la S.V.E.P.

Démonstration. Remarquons que si $T$ est hyponormal alors $\forall \lambda \in \mathbb{C}, \lambda I-T$ est hyponormal, d'autre part $\lambda I-T$ hyponormal $\Rightarrow H_{0}(\lambda I-T)=N(\lambda I-T)$ (voir [15, Lemme 3.1.5]) donc $\forall \lambda \in \mathbb{C}, H_{0}(\lambda I-T)$ est fermé et d'après la proposition précédente, on en déduit que $T$ a la S.V.E.P.

Proposition 1.12. Soit $A$ un opérateur fermé avec $D(A)$ dense dans $X$ et $\lambda_{0} \in \mathbb{C}$ tels que $R\left(\lambda_{0} I-A\right)$ soit fermé et $N\left(\lambda_{0} I-A\right)=\{0\}$. Alors on $a$ :

(1) $A$ a la S.V.E.P. en $\lambda_{0}$.

(2) Si en plus $D(A)$ est dense dans $X$ et $R\left(\lambda_{0} I-A\right) \neq X$ alors $A^{*}$ n'a pas la S.V.E.P. 
Démonstration. (1) Se déduit directement du [16, Théorème 1.4].

(2) $R\left(\lambda_{0} I-A\right)$ fermé et $N\left(\lambda_{0} I-A\right)=\{0\} \Rightarrow R\left(\bar{\lambda}_{0} I-A^{*}\right)=X^{*}$, d'autre part

$$
R\left(\lambda_{0} I-A\right) \neq X \Rightarrow N\left(\bar{\lambda}_{0} I-A^{*}\right) \neq\{0\} .
$$

Du Corollaire 1.6 on déduit la fin de la démonstration.

Remarque. L'exemple 1.7 [4] se déduit directement de la Proposition 1.12.

"Soit $T$ un opérateur borné dans un espace de Hilbert H. Si $T$ est une isométrie non-unitaire, alors $T^{*}$ n'a pas la S.V.E.P."

En effect $T$ isométrie $\Rightarrow N(T)=\{0\}$ et $R(T)$ fermé et comme elle est non unitaire, $R(T) \neq H$. D'après le (2) de la Proposition 1.12 on en déduit que $T^{*}$ n'a pas la S.V.E.P.

\section{LES OPÉRATEURS DONT LE COEUR ANALYTIQUE EST RÉDUIT À ZÉRO}

Dans la suite $H$ désigne un espace de Hilbert et $B(H)$ l'espace de tous les opérateurs bornés.

Notons $\mathscr{K} \mathscr{A}_{0}(H)=\{T \in B(H) ; K(T)=\{0\}\}$.

Exemples. (1) $\mathscr{Q}_{\mathrm{af}} \subseteq \mathscr{R} \mathscr{A}_{0}(H)$ (voir Remarque 1.1(b)).

(2) $H=l^{2}$ et $\left\{e_{n}\right\}_{n \geq o}$ est la base canonique de $l^{2}$, on définit $T$ par $T e_{n}=$ $e_{n+1}$ alors $\cap_{n \geq 0} R\left(T^{n}\right)=\{0\}$ donc $K(T)=\{0\}$; c'est à dire $T \in \mathscr{R} \mathscr{A}_{0}(H)$.

(3) Crownover [6] étudie les opérateurs $T$ tels que $N(T)=\{0\}, \operatorname{codim}(R(T))$ $=1$ et $\cap_{n \geq 0} R\left(T^{n}\right)=\{0\}$. Un tel opérateur $T$ est dans $\mathscr{K} \mathscr{A}_{0}(H)$.

(4) $\mathrm{Si} \bar{T} \in B_{n}(\omega)$ l'ensemble des opérateurs de Cowen-Douglas [5] alors, $0 \in \omega \Rightarrow T^{*} \in \mathscr{K}_{0}(H)$ (voir [15, Théorème 2.3.6]).

Proposition 2.1. Si $T \in \mathscr{K} \mathscr{A}_{0}(H)$, alors $0 \in \sigma\left(T_{\mid M}\right)$ pour tout sous-espace invariant $M \neq\{0\}$. En particulier, $\sigma_{p}(T) \subseteq\{0\}$ et $\sigma(T)$ est connexe et contient zéro.

Démonstration. Si $T M \subseteq M(M \neq\{0\})$ et $T_{\mid M}$ est inversible, alors pour tout $u \in M$ et tout $n \geq 0$, on a $u=T^{n}\left(\left(T_{\mid M}\right)^{-n} u\right)$ et $v_{n}=\left(T_{\mid M}\right)^{-n} u$ satisfait $\left\|v_{n}\right\| \leq\left\|\left(T_{\mid M}\right)^{-1}\right\|^{n}\|u\|$ donc $M \subseteq K(T)=\{0\}$, d'où la contradiction. Les autres résultats se déduisent immediatement.

Cette proposition nous donne quelques corollaires.

Corollaire 2.2. Soit $T \in \mathscr{K} \mathscr{A}_{0}(H)$. Alors:

(i) 0 est isolé dans $\sigma(T) \Leftrightarrow T$ quasi-nilpotent.

(ii) $T$ est décomposable $\Leftrightarrow T$ quasi-nilpotent.

(iii) $T$ est normal $\Leftrightarrow T=0$.

Corollaire 2.3. [[10, Corollaire 3.4]] Soit $T \in B(H)$ alors $T \in \mathscr{Q}_{\text {af }}$ et décomposable $\Leftrightarrow T$ quasi-nilpotent.

Proposition 2.4. (cf. [16, Proposition 1.8]). Soit $T \in B(H)$ alors $H_{0}(T) \subseteq$ $K\left(T^{*}\right)^{\perp}$. 
Corollaire 2.5. Soit $T \in B(H)$ alors $\overline{H_{0}(T)}=H$ et 0 est isolé dans $\sigma(T) \Leftrightarrow T$ quasi-nilpotent.

Démonstration. La Proposition $2.4 \Rightarrow K\left(T^{*}\right) \subseteq H_{0}(T)^{\perp}=\{0\} \Rightarrow K\left(T^{*}\right)=$ $\{0\}$. 0 est isolé $\sigma(T) \Rightarrow 0$ est isolé dans $\sigma\left(T^{*}\right)$ et d'après le Corollaire 2.2, on en déduit que $T^{*}$ est quasi-nilpotent et donc $T$ est quasi-nilpotent. La réciproque est évidente.

Remarque. Le Corollaire 2.5 a été démontré dans [8, Théorème 2], d'une façon différente.

Notons. $\sigma_{\omega}(T):=\{\lambda \in \mathbb{C} ; \lambda I-T$ n'est pas de Fredholm d'indice 0$\} . \sigma_{\omega}(T)$ est appelé spectre de Weyl de $T$.

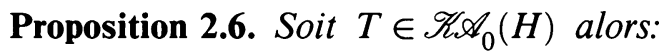

(1) $T$ a la S.V.E.P.

(2) $\sigma(T)=\sigma_{\omega}(T)$.

Démonstration. (1) $T \in \mathscr{K} \mathscr{A}_{0}(H) \Rightarrow \forall \lambda \neq 0, N(\lambda I-T) \subseteq K(T)=\{0\}$, donc $N(\lambda I-T)=\{0\}$; ou encore, $\sigma_{p}(T) \subseteq\{0\}$, d'où $\sigma_{p}(T)$ a un intérieur vide et par conséquent $T$ a la S.V.E.P.

(2) Il suffit de montrer $\sigma(T) \subseteq \sigma_{\omega}(T)$. Si $\lambda \notin \sigma_{\omega}(T)$ alors $\lambda I-T$ est de Fredholm d'indice nul.

Si $\lambda \neq 0$ alors $N(\lambda I-T)=\{0\}$ et comme l'indice est nul on en déduit que $N\left(\overline{\lambda I}-T^{*}\right)=\{0\}$ donc $R(\lambda I-T)$ est dense dans $H$, or $\lambda I-T$ est Fredholm donc $R(\lambda I-T)$ est fermé donc $\lambda \in \rho(T)$ donc $\lambda \notin \sigma(T)$.

Si $\lambda=0, K(T)=\{0\}$ implique $0 \in \sigma(T)$, montrons que $0 \in \sigma_{\omega}(T)$. Supposons le contraire et comme $\rho_{\omega}(T)=\mathbb{C} \backslash \sigma_{\omega}(T)$ est ouvert, on en déduit (comme plus haut) que 0 est isolé dans le spectre de $T$. D'après le Corollaire 2.2 on a $\sigma(T)=\{0\}$ donc $T$ est quasi-nilpotent et Fredholm, par conséquent (voir [16, Corollaire 2.15]) $T$ est nilpotent et de Fredholm, par conséquent $\operatorname{dim} H<\infty$, d'où la contradiction, donc $0 \in \sigma_{\omega}(T)$.

Lemme 2.7. Soient $T$ et $S$ deux opérateurs de $B(H)$ tels que $T$ et $S$ soient quasi-similaires alors $K(T)=\{0\} \Leftrightarrow K(S)=\{0\}$.

Démonstration. $T$ et $S$ étant quasi-similaires, par définition $\exists X, Y$ deux opérateurs quasi-inversibles (i.e., injectifs et à images denses dans $H$ ) tels que $X T=S X$ et $T Y=Y S$, d'où on en déduit que $\forall n \geq 0, X T^{n}=S^{n} X$ et $T^{n} Y=Y S^{n}$, de là, on montre facilement que $X(K(S)) \subseteq K(T)$ et $Y(K(T)) \subseteq$ $K(S)$. $X$ et $Y$ étant injectifs, on en déduit le lemme.

Corollaire 2.8. Soit $T \in \mathscr{K} \mathscr{A}_{0}(H)$ alors $\{S \in B(H) ; S$ est quasi-similaire à $T\}$ $\subseteq \mathscr{K} \mathscr{A}_{0}(H)$.

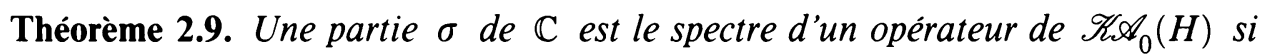
et seulement si $\sigma$ est compact, connexe et contient zéro.

Démonstration. "Seulement si" se déduit de la Propositon 2.1. 
"Si." $\sigma$ est compact, connexe et contient zéro, d'après [9, Théorème 3.2] il existe $T \in B(H)$ tel que $T$ soit une somme directe d'opérateurs nilpotents avec $\sigma(T)=\sigma$. On en déduit que $K(T)=\{0\}$ et donc $T \in \mathscr{H} \mathscr{\bigotimes}_{0}(H)$.

Remarque. Ce dernier théorème généralise [10, Théorème 3.11].

\section{SUR LES LIMITES D'OPÉRATEURS NILPOTENTS}

Dans cette section, nour donnons une condition suffisante pour qu'un opérateur soit dans l'adhérence des nilpotents.

Tout d'abord quelques notations:

$$
\begin{aligned}
& \rho_{S \Phi}(T)=\{\lambda \in \mathbb{C} ; \lambda I-T \text { est semi-Fredholm }\} \\
& \sigma_{\Phi}(T)=\{\lambda \in \mathbb{C} ; \lambda I-T \text { n'est pas Fredholm }\} \\
& \mathscr{N}=\{\text { des opérateurs nilpotents }\} . \\
& q N=\{\text { des opérateurs quasi-nilpotents }\} \\
& Q_{\text {af }}=\left\{T \in B(H) ; T \text { et } T^{*} \in \mathscr{Q}_{\text {af }}\right\}=\mathscr{Q}_{\text {af }} \cap \mathscr{Q}_{\text {af }}^{*} .
\end{aligned}
$$

Notons. Aussi $\mathscr{H} \mathscr{A}(H)=\left\{T \in B(H) ; K(T)=K\left(T^{*}\right)=\{0\}\right\}$.

Remarques.

$$
\mathscr{N} \subsetneq q N \subsetneq Q_{\mathrm{af}} \subseteq \mathscr{H} \mathscr{A}(H) \subseteq \mathscr{H} \mathscr{A}_{0}(H) .
$$

La dernière inclusion est stricte, car l'opérateur $T$ de l'exemple (2), §2, appar-

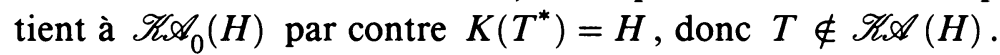

Conjecture. $Q_{\mathrm{af}}=\mathscr{H} \mathscr{A}(H)$.

Remarques. (1) D'après le théorème d'Apostol [1]:

$$
\overline{H_{0}(T)}=\overline{H_{0}\left(T^{*}\right)}=H \Leftrightarrow T \in Q_{\mathrm{af}} .
$$

Il est clair que, si l'inclusion inverse de la Proposition 2.3 [16, Question 1] est vérifiée alors $\mathscr{H} \mathscr{A}(H)=Q_{\mathrm{af}}$.

(2) Le Corollaire 2.2(ii) et le Corollaire 2.3 impliquent que la conjecture est vérifiée dans le cas des opérateurs décomposables.

(3) Dans $\S 4$, nous démontrons la conjecture dans le cas des opérateurs pseudoFredholm (Théorème 4.3).

Proposition 3.1. Soit $T$ un opérateur bitriangulaire (cf. $[14,7])$ alors $d(T)=0$ si et seulement si $T \in \mathscr{K} \mathscr{A}(H)$ (où $d(T)$ est la diagonale de $T$ ).

Démonstration. Si: $T \in \mathscr{H} \mathscr{A}(H)$, d'après la Proposition 2.1, $\sigma_{p}(T) \subseteq\{0\}$. Or d'après [7, Théorème 3.1], si $T$ est bitriangulaire alors $d(T)=d\left(T^{*}\right)^{*}=$ $\sigma_{p}(T) \subseteq\{0\}$, d'où $d(T)=0$.

Seulement si: Si $T$ bitriangulaire avec $d(T)=0$, alors [7, Théorème 4.6] implique que $T$ est quasi-similaire à un opérateur dont le coeur analytique est réduit à zéro. Le Corollaire 2.8 permet de conclure.

Théorème 3.2. Soit $T \in \mathscr{H} \mathscr{A}(H)$ alors:

(1) $\sigma(T)$ est connexe et contient zéro,

(2) $\forall \lambda \in \sigma_{\mathrm{S} \Phi}(T), \operatorname{Ind}(\lambda I-T)=0$, 
(3) $\sigma_{\Phi}(T)=\sigma(T)$,

(4) $\sigma_{p}(T) \subseteq\{0\}$,

(5) $\sigma_{r}(T) \subseteq\{0\}$,

(6) $\sigma(T)=\sigma_{c}(T) \cup\{0\}$,

où $\sigma_{c}(T)=\{\lambda \in \mathbb{C} ; \lambda I-T$ est injectif, non surjectif et $\overline{R(\lambda I-T)}=H\} \quad$ (le spectre continu de $T)$.

Démonstration. (1) et (4) se déduisent de la Proposition 2.1 et du fait que

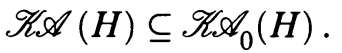

(2) Remarquons que $\forall \lambda \neq 0, N(\lambda I-T) \subseteq K(T)$ et $N\left(\overline{\lambda I}-T^{*}\right) \subseteq K\left(T^{*}\right)$. Donc si $T \in \mathscr{H} \mathscr{A}(H)$ alors $\forall \lambda \neq 0, N(\lambda I-T)=N\left(\overline{\lambda I}-T^{*}\right)=\{0\}$, d'où $\forall \lambda \neq 0$, $\operatorname{Ind}(\lambda I-T)=0$. Si $\lambda=0$, supposons que $0 \in \rho_{\mathrm{S} \Phi}(T)$, comme $\rho_{\mathrm{S} \Phi}(T)$ est ouvert, on en déduit qu'il existe $\mathscr{U}_{0}$ voisinage de zéro dans $\mathbb{C}$ tel que $\mathscr{U}_{0} \subseteq \rho_{\mathrm{S} \Phi}(T)$ donc $\forall \lambda \in \mathscr{U}_{0}-\{0\}$, on a $N(\lambda I-T)=N\left(\overline{\lambda I}-T^{*}\right)=\{0\}$ et $R(\lambda I-T)$ est fermé, par conséquent $\mathscr{U}_{0}-\{0\} \subseteq \rho(T)$ donc 0 est isolé dans $\sigma(T)$ et d'après le Corollaire 2.2 on a $\sigma(T)=\{0\}$ donc $T$ est quasi-nilpotent et semi-Fredholm, d'après [16, Corollaire 2.15], on en déduit que $T$ est nilpotent et semi-Fredholm donc $\operatorname{dim} H<\infty$, d'où la contradiction. Donc $0 \notin \rho_{\mathrm{S} \Phi}(T)$ par conséquent $\forall \lambda \in \rho_{\mathrm{S} \Phi}(T), \operatorname{Ind}(\lambda I-T)=0$.

(3) on utilise les mêmes arguments que pour (2).

(5) et (6) faciles à vérifier.

Théorème 3.3. (Apostol - Foïas - Voiculescu [13, Théorème 5.1, p. 108]) . L'adhérence de l'ensemble des opérateurs nilpotents coïncide avec l'ensemble des opérateurs $T \in B(H)$ qui vérifient les conditions suivantes:

(i) $\sigma(T)$ est connexe et contient zéro.

(ii) $\sigma_{\Phi}(T)$ est connexe et contient zéro.

(iii) $\operatorname{Ind}(\lambda I-T)=0 \forall \lambda \in \rho_{\mathrm{S} \Phi}(T)$.

Corollaire 3.4. (1) $\mathscr{K} \mathscr{A}(H) \subsetneq \bar{N}$,

(2) $\overline{\mathscr{K} \mathscr{A}(H)}=\overline{\mathscr{N}}$.

Démonstration. (1) L'inclusion se déduit du Théorème 3.2(1), (2), (3) et du Théorème 3.3. Cette inclusion est stricte car Herrero [13, p. 108], démontre qu'il existe des opérateurs normaux non nuls et qui sont dans l'adhérence des opérateurs nilpotents. Par contre d'après le Corollaire 2.2(iii), si $T$ est normal et $K(T)=\{0\}$ alors $T=0$.

(2) Se déduit du fait que $\mathscr{K} \mathscr{A}(H)$ contient les nilpotents et de (1).

Remarque. Du Corollaire 3.4 on déduit que $\overline{Q_{\text {af }}}=\overline{\mathscr{N}}$.

Théorème 3.5. Soit $T$ dans l'adhérence des nilpotents $(\overline{\mathcal{N}})$ et $\varepsilon>0$, alors il existe $K_{\varepsilon}$ opérateur compact avec $\left\|K_{\varepsilon}\right\| \leq \varepsilon$ tel que $T+K_{\varepsilon} \in \mathscr{H} \mathscr{A}(H)$.

Démonstration. Si $T \in \bar{N}(\subseteq(B Q T)$ [13, Théorème 6.17, p. 153]) et $\varepsilon>0$, alors (d'après [14]) il existe $K_{\varepsilon}$ opérateur compact avec $\left\|K_{\varepsilon}\right\| \leq \varepsilon$ tel que $T+K_{\varepsilon}$ soit bitriangulaire avec diagonale nulle, la Proposition 3.1 permet de conclure que $T+K_{\varepsilon} \in \mathscr{H} \mathscr{A}(H)$. 


\section{CAS DES OPÉRATEURS PSEUdo-FrEDHOLM}

Définition 4.1. Soit $A$ un opérateur fermé. A est dit pseudo-Fredholm (et on note $A \in \mathrm{P} \Phi)$, s'il existe $M, N$ deux sous-espaces fermés de $H$ vérifiant:

(a) $H=M \oplus N$.

(b) $A(M \cap D(A)) \subseteq M$ et si on note $A_{0}=A_{\mid M}$ alors $\forall n \geq 0, N\left(A_{0}^{n}\right) \subseteq$ $R\left(A_{0}\right), R\left(A_{0}\right)$ étant fermé.

(c) $N \subseteq D(A), A(N) \subseteq N$ et $A_{\mid N}$ est quasi-nilpotent.

Remarque. Pour les propriétés des opérateurs pseudo-Fredholm voir [17, 18].

Il est clair que si $A$ n'a pas la S.V.E.P., alors l'intérieur de $\sigma_{p}(A)$ est non vide. La réciproque en géneral n'est pas vraie. Le théorème suivant montre qu'elle l'est dans le cas des opérateurs pseudo-Fredholm.

Théorème 4.2. Soit $A$ un opérateur fermé et $\mu \in \mathbb{C}$, tels que $A-\mu I \in \mathrm{P} \Phi$.

Si $\mu$ est à l'intérieur de $\sigma_{p}(A)$ alors $A$ n'a pas la S.V.E.P.

Démonstration. Si $\mu$ est à l'intérieur de $\sigma_{p}(A)$ alors $\exists \mathscr{U}$ ouvert de $\mathbb{C}$, inclus dans $\sigma_{p}(A)$ qui contient $\mu$. On a $\forall \lambda \in \mathscr{U}-\{\mu\}, N(A-\lambda I) \subseteq K(A-\mu I)$. En outre $A-\mu I \in \mathrm{P} \Phi$ implique que $K(A-\mu I)$ est fermé (cf. [18, Lemme 2.1]). D'où on en déduit d'une part que $K(A-\mu I) \neq\{0\}$ et d'autre part que $\mathscr{U}-\{\mu\} \subseteq \sigma_{p}\left(A_{\mid K(A-\mu I)}\right)$; comme $\sigma\left(A_{\mid K(A-\mu I)}\right)$ est fermé on en déduit que $\mu \in \sigma\left(A_{\mid K(A-\mu I)}\right)$, or $A-\mu I$ est surjectif sur $K(A-\mu I)$ par conséquent $N\left(A-\mu I_{\mid K(A-\mu I)}\right) \neq\{0\}$ et $N\left(A-\mu I_{\mid K(A-\mu I)}\right) \subseteq K(A-\mu I) \cap H_{0}(A-\mu I) \neq\{0\}$, d'après le Corollaire 1.5 , on conclue que $A$ n'a pas la S.V.E.P.

Théorème 4.3. Soit $T$ borné et $T \in \mathrm{P} \Phi$. Alors les quatre conditions suivantes sont équivalentes:

(1) $T \in \mathscr{R} \mathscr{A}(H)$.

(2) $\overline{H_{0}(T)}=\overline{H_{0}\left(T^{*}\right)}=H$.

(3) $T \in \mathscr{Q}_{\text {af }} \cap \mathscr{Q}_{\text {af }}^{*}$.

(4) $T \in q N$.

Démonstration. (1) $\Leftrightarrow$ (2) se déduit de [18, Proposition 2.4].

(2) $\Leftrightarrow$ (3) Voir [1].

(3) $\Rightarrow$ (4) Supposons que $T \in \mathrm{P} \Phi \cap\left[\mathscr{Q}_{\mathrm{af}} \cap \mathscr{Q}_{\mathrm{af}}^{*}\right]$ et $M, N$ comme dans la Définition 4.1 alors $H=M \oplus N$ et $N \subseteq H_{0}(T)$, impliquent que $H_{0}(T)=$ $H_{0}(T) \cap M+N=H_{0}\left(T_{\mid M}\right)+N$. D'après [18, Remarque 1.3(e)] on a $H_{0}\left(T_{\mid M}\right) \subseteq$ $K\left(T_{\mid M}\right) \subseteq K(T)=\{0\}$ d'où $H_{0}(T)=N$. La Proposition 2.4, [18], implique que $N^{\perp}=H_{0}(T)^{\perp}=K\left(T^{*}\right)=\{0\}$ d'où $N=H_{0}(T)=H$, la Remarque 1.1(c) permet alors de conclure que $T \in q N$.

(4) $\Rightarrow$ (3) Évident.

Corollaire 4.4. Si $\operatorname{dim}(H)=\infty$ alors

$$
\mathrm{S} \Phi \cap q N=\mathrm{S} \Phi \cap\left[\mathscr{Q}_{\mathrm{af}} \cap \mathscr{Q}_{\mathrm{af}}^{*}\right]=\mathrm{S} \Phi \cap \mathscr{K} \mathscr{A}(H)=\varnothing
$$

Où S $\Phi$ dénote l'ensemble des opérateurs semi-Fredholm. 


\section{ACKNOWLEDGMENT}

L'auteur tiens à remercier D. A. Herrero qui a bien voulu lire le manuscrit de ce travail et qui a suggéré de nombreuses améliorations de sa rédaction.

\section{REFERENCES}

1. C. Apostol, Quasi-affine transforms of quasinilpotent compact operators, Rev. Roumaine Math. Pures Appl. 21 (1976), 813-816.

2. C. Apostol, C. Foias, et D. Voiculescu, on the norm closure of nilpotents. II, Rev. Roumaine Math. Pures Appl. 19 (1974), 549-557.

3. __ Some results on non-quasitriangular operators. IV, Rev. Roumaine Math. Pures Appl. 18 (1973), 487-514.

4. I. Colojoara et C. Foias, Theory of generalized spectral operators, Gordon and Breach, New York, 1968.

5. M. J. Cowen et R. G. Douglas, Complex geometry on operators theory, Bull. Amer. Math. Soc. 83 (1977), 131-133.

6. R. M. Crownover, Commutants of shifts on Banach spaces, Michigan Math. J. 19 (1972), 233-247.

7. K. R. Davidson et D. A. Herrero, The Jordan form of bitriangular operator, preprint.

8. I. Erdelyi et F. R. Miller, Decomposition theorems for partial isometries, J. Math. Anal. Appl. 3o (1970), 665-679.

9. L. A. Fialkow, Direct sums of quasi-nilpotents operators, Proc. Amer. Math. Soc. 48 (1975), 125-131.

10. _ A note on quasi-similarity of operators, Acta. Sci. Math. 39 (1977), 67-85.

11. _ A note on quasi-similarity. II, Pacific J. Math. 70 (1977), 151-162.

12. J. K. Finch, The single valued extension property on a Banach space, Pacific J. Math. 58 (1975), 61-69.

13. D. A. Herrero, Approximation of Hilbert spaces operators. I, vol. 72, Pitman, London, Boston, and Melbourne, 1982.

14. __ Most quasitriangular operators are triangular, most biquasitriangular operators are bitriangular, J. Operator Theory (à paraître).

15. M. Mbekhta, Généralisation de la décomposition de Kato aux opérateurs paranormaux et spectraux, Thèse de 3ème cycle, Université de Nice, 1984.

16. __ Généralisation de la décomposition de Kato aux opérateurs paranormaux et spectraux, Glasgow Math. J. 29 (1987), pp. 159-175.

17. __ Décomposition de Kato généralisée, C. R. Acad. Sci. Paris, Sér. I Math. 33 (1986).

18. __ Sur l'unicité de la décomposition de Kato généralisée, Acta. Sci. Math. (à paraître).

19. F. H. Vasilescu, Analytic functional calculus, and spectral decompositions, Reidel, 1982.

20. P. Vrbova, On local spectral properties of operators in Banach spaces, Czechoslovak Math. J. 23 (1973), 483-492.

Département de Mathématiques, Université de Nice, Parc Valrose, F-06034 Nice, Cedex, France Cedex

Current address: U.F.R. de Mathématiques, Université de Lille I, F-59655 Villeneuve d'Ascq 